\title{
COVID-19 in an Asymptomatic Renal Transplant Recipient Employed in the Health Care Setting: A Case Report
}

\author{
Harsha Adnani, MBBS ${ }^{1}$ Akshay Khatri, MBBS, MD² Nirav Agrawal, MBBS ${ }^{1}$ \\ Ernesto Molmenti, MD, PhD, MBA ${ }^{3}$ Madhu Bhaskaran, MD ${ }^{4}$
}

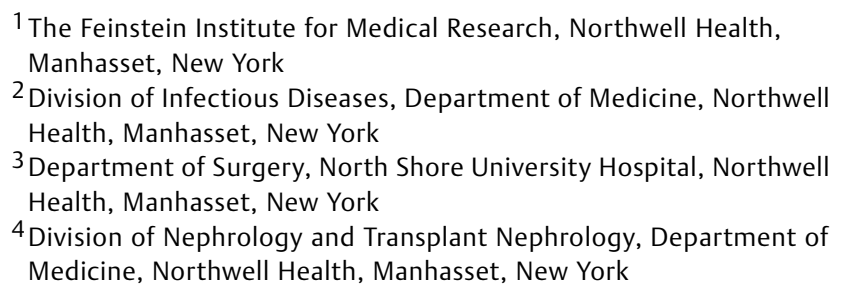

Address for correspondence Harsha Adnani, MBBS, The Feinstein Institute for Medical Research, Northwell Health, 350 Community Dr, Manhasset, NY 11030 (e-mail: hadnani@northwell.edu).

Int J Angiol 2022;31:289-291.

\begin{abstract}
Keywords

- COVID-19

- asymptomatic

- immunosuppressed

- public health

- health care worker

During the ongoing pandemic, there have been varying presentations of coronavirus disease 2019 (COVID-19) infection, with the concern that patients who are immunosuppressed (due to underlying medical conditions and/or therapies) are at higher risk of severe disease. We report the case of an elderly renal transplant recipient working in a long-term health care facility who was being monitored by weekly surveillance testing and tested positive for COVID-19 by polymerase chain reaction (PCR) testing, despite having no clinical symptoms. He recovered with supportive care, despite being on multiple long-term immunosuppressant drugs and having multiple comorbidities. Additionally, it was found that he did not mount an antibody response, when he tested negative by serologic testing. Through this case, we wish to highlight the unique clinical scenario of asymptomatic patients who may have an underwhelming immune response to COVID-19, but may nevertheless be an important source of dissemination. We further discuss the probable mechanism of such asymptomatic presentations in immunosuppressed patients, while reinforcing the importance of self-isolation of COVID-19 patients (particularly in asymptomatic health care workers).
\end{abstract}

The coronavirus disease 2019 (COVID-19) pandemic, caused by spread of the severe acute respiratory syndrome coronavirus-2 (SARS-CoV-2), has become the most important global health crisis in modern times. There have been severe clinical manifestations reported with COVID-19, although these may not be seen in a similar manner in patients with immunosuppressive conditions or those on immunosuppressive medications. ${ }^{1-3}$ According to the Centers for Disease Control and Prevention (CDC), immunocompromised individuals such as solid organ transplant recipients are considered at increased risk for severe illness from COVID19, regardless of age. ${ }^{4}$ COVID-19 may present as a milder form of infection in the immunosuppressed patients, leading to underdiagnosis. ${ }^{5}$ Health care workers (HCWs) are prone to acquiring COVID-19. ${ }^{6}$ At present, there is insufficient literature available on COVID-19 in asymptomatic immunosuppressed HCWs. We present a case of an asymptomatic transplant recipient working in the health care setting who tested positive for COVID-19 during surveillance testing. article published online

March 11, 2021 (c) 2021. International College of Angiology. All rights reserved. Thieme Medical Publishers, Inc., 333 Seventh Avenue, 18th Floor, New York, NY 10001, USA
DOI https://doi.org/ 10.1055/s-0041-1725183. ISSN 1061-1711. 


\section{Case Report}

A 67-year-old man who is a living related-donor kidney recipient presented to the employee health clinic of our hospital for his routine weekly state-mandated COVID-19 testing. He worked as a food aide in a long-term care facility, but due to the ongoing pandemic and his immunosuppressed status his work duties were switched to avoid direct patient contact. His laboratory testing was unremarkable except for a positive result on the COVID-19 nasopharyngeal swab by reverse transcriptase polymerase chain reaction (rtPCR) testing. He reported the result to his transplant nephrologist who follows him locally and was placed on home quarantine.

His past medical history is significant for gout, end-stage renal disease on hemodialysis from chronic glomerulonephritis (immunoglobulin A [IgA] nephropathy), hyperlipidemia, and prediabetes. He received a kidney transplant from (family member/relation) outside the United States 4 years prior and has had stable allograft function. He was born in Southern India and migrated to the United States $\sim 20$ years back. His current medication regimen includes metformin, mycophenolate mofetil $360 \mathrm{mg}$ twice daily; prednisone $5 \mathrm{mg}$ once daily; tacrolimus $3 \mathrm{mg}$ in the morning and $2 \mathrm{mg}$ in the evening; trimethoprim-sulfamethoxazole single-strength tablet daily; and vitamin D3. He denied any upper respiratory symptoms, recent travel, or sick contacts. His vitals are stable. No chest imaging was performed. No recent changes were made to his immunosuppressive medications.

Immediately after the positive rtPCR result, he was placed on home isolation for 14 days. Education on health of the patient and precautions for family members was provided by the employee health services. He remained symptom-free after 2 weeks of twice-weekly monitoring through telemedicine consultations, with none of his family members developing any symptoms. He resumed his position after a negative rtPCR result and none of his coworkers were subsequently diagnosed with COVID-19. Seven weeks after his positive rtPCR, his COVID-19 immunoglobulin G (IgG) antibody testing was performed and was surprisingly negative. He remains COVID-19 rtPCR negative on weekly surveillance testing and continues to follow closely with his outpatient transplant nephrologist.

\section{Discussion}

We performed a literature search online (PubMed, Medline) from February 1, 2020, to July 25, 2020, with the search/MESH criteria "asymptomatic, COVID-19 positive, immunocompromised, healthcare worker." Our inclusion criteria were an asymptomatic COVID-19 positive HCW who is immunosuppressed. We excluded any symptomatic COVID-19 patients. Additionally, we only regarded the studies published in English. There were no articles found matching our inclusion criteria. Interestingly in a recent retrospective study among end-stage renal disease patients undergoing dialysis therapy, 44 of 235 patients were reported to be asymptomatic with positive SARS-CoV-2 antibody testing. ${ }^{7}$ Thus, we believe that our patient may be one of the first cases of an asymptomatic immunosuppressed HCW who tested positive for COVID-19.

Patients with COVID-19 often present with symptoms such as fever, cough, and dyspnea. The poor prognostic indicators that are increasingly being associated with COVID-19 include older age; male sex; and comorbidities like diabetes, obesity, and cardiovascular disease. ${ }^{1}$ Immunosuppressed patients may remain underdiagnosed, especially if presenting as a mild illness. Consequently, they become an important source for viral shedding. Qi et $\mathrm{al}^{8}$ studied the duration and factors associated with viral shedding in COVID-19-positive patients. They found that patients on immunosuppressant drugs like glucocorticoids had significantly prolonged viral shedding of more than the median of 17 days. Viral shedding has been reported to be the highest before symptom onset, suggesting high transmissibility before clinical identification of the disease. ${ }^{9}$ Thus, testing and identifying asymptomatic immunosuppressed patients is essential to break the chain of transmission.

Immunosuppresants are known to alter humoral and cellmediated immunity and affect neutrophil function, increasing the risk of certain viral infections like influenza and rhinovirus. $^{5}$ However, this does not appear to occur in human coronavirus infections-earlier outbreaks due to severe acute respiratory syndrome coronavirus (SARS-CoV) and Middle East respiratory syndrome coronavirus (MERS-CoV) did not show any change in incidence of infections or change in prognosis in immunocompromised patients. ${ }^{5}$ SARS-CoV-2 is believed to produce an intense immune response ("cytokine storm") that results in its diverse and severe clinical manifestations. ${ }^{10}$ Thus, the weaker immune response (as that which may occur in immunocompromised patients), may exert a protective effect and lead to a milder disease course. ${ }^{5}$ However, this observation has not been consistently reported in renal transplant recipients-morbidity from severe disease and mortality have been reported in hospitalized patients. ${ }^{3}$

According to the CDC, many individuals infected with COVID-19 may take a longer time to mount an antibody response and some may never develop antibodies. ${ }^{11}$ It is also known that it can take up to 1 to 3 weeks to establish antibodies. Clarke et al found that 2 out of 79 patients who were on immunosuppression that had rtPCR-positive results but did not have detectable antibodies. ${ }^{6}$ Our patient had a positive COVID-19 rtPCR test, with negative antibody test, in line with these observations.

Public health and safety are significant concerns when resuming work during a pandemic. Zhao et al ${ }^{12}$ showed that asymptomatic COVID-19 patients could act as carriers and transmit the infection as efficiently as symptomatic cases. It is imperative to follow the current state and institutional guidelines of isolation (currently mandated at 10 days in New York state), to break the chain of transmission in those with positive rtPCR testing. ${ }^{13}$ The recent observation of prolonged viremia in the bloodstream of blood donors who recovered from COVID-19 is another concerning finding that highlights the potential risk of asymptomatic spread. ${ }^{14}$

Health care institutions have taken several measures to keep HCWs safe and prevent onward transmission. 
Mandatory weekly testing of the HCW via real-time rtPCR is being done in long-term care facilities. ${ }^{15}$ Temperature and symptom screening are done routinely and anyone with symptoms is advised to stay at home and self-isolate. Studies have shown that weekly testing can prevent 16 to $33 \%$ onward transmission from HCWs, depending on the availability of the test results. ${ }^{16}$

\section{Conclusion}

Immunosuppressed individuals-those on immunosuppressive therapy after a solid organ transplant, or as part of therapy against cancer and autoimmune diseases-should be under close surveillance for COVID-19 infection. Some patients have been shown to have poor outcomes, while others are found to be asymptomatic, yet posing the risk of community spread. Early diagnosis and isolation of asymptomatic cases is important to prevent transmission. Further studies are needed to understand the specific factors that affect disease severity and those that have important transmission implications in this vulnerable population.

\section{Conflict of Interest}

None declared.

\section{Acknowledgments}

None.

\section{References}

1 Huang C, Wang Y, Li X, et al. Clinical features of patients infected with 2019 novel coronavirus in Wuhan, China. Lancet 2020;395 (10223):497-506

2 Vizcarra P, Pérez-Elías MJ, Quereda C, et al; COVID-19 ID Team. Description of COVID-19 in HIV-infected individuals: a singlecentre, prospective cohort. Lancet HIV 2020;7(08):e554-e564

3 Nair V, Jandovitz N, Hirsch JS, et al. COVID-19 in kidney transplant recipients. Am J Transplant 2020;20(07):1819-1825

4 Centers for Disease Control and Prevention Coronavirus disease. 2019(COVID-19):pPeople with certain medical conditions. https:// www.cdc.gov/coronavirus/2019-ncov/need-extra-precautions/
people-with-medicalconditions.html?CDC_AA_refVal=https\%3A\% 2F\%2Fwww.cdc.gov\%2Fcoronavirus\%2F2019-ncov\%2Fneed-extraprecautions\%2Fgroups-at-higher-risk.html. Accessed July 26, 2020

5 D'Antiga L. Coronaviruses and immunosuppressed patients: the facts during the third epidemic. Liver Transpl 2020;26(06): 832-834

6 Nguyen LH, Drew DA, Joshi AD, et al. Risk of COVID-19 among frontline healthcare workers and the general community: a prospective cohort study. Preprint. Medrxiv 2020

7 Clarke C, Prendecki M, Dhutia A, et al. High prevalence of asymptomatic COVID-19 infection in hemodialysis patients detected using serologic screening. J Am Soc Nephrol 2020;31 (09):1969-1975

8 Qi L, Yang Y, Jiang D, et al. Factors associated with the duration of viral shedding in adults with COVID-19 outside of Wuhan, China: a retrospective cohort study. Int J Infect Dis 2020;96:531-537

9 Huff HV, Singh A. Asymptomatic transmission during the COVID19 pandemic and implications for public health strategies. Clin Infect Dis 2020:ciaa654

10 Mehta P, McAuley DF, Brown M, Sanchez E, Tattersall RS, Manson JJHLH Across Speciality Collaboration, UK. COVID-19: consider cytokine storm syndromes and immunosuppression. Lancet 2020;395(10229):1033-1034

11 Centers for Diseases Control and Prevention Coronavirus disease. 2019(COVID-19): test for past infection. https://www.cdc.gov/coronavirus/2019-ncov/testing/serology-overview.html Accessed August 3, 2020

12 Zhao H, Lu X, Deng Y, Tang Y, Lu J. COVID-19: asymptomatic carrier transmission is an underestimated problem. Epidemiol Infect 2020;148:e116

13 Centers for Diseases Control and Prevention. https://www.cdc. gov/coronavirus/2019-ncov/hcp/duration-isolation.html. Accessed August 19, 2020

14 Pham TD, Huang C, Wirz OF, et al. SARS-CoV-2 RNAemia in a healthy blood donor 40 days after respiratory illness resolution. Ann Intern Med 2020;173(10):853-854

15 Department of health, New York State Department of Health. Required COVID-19 testing for all nursing home and adult care facility personnel. https://www.health.ny.gov/professionals/hospital_administrator/letters/2020/docs/dal_20-14_covid_required_ testing.pdf. Published May 11, 2020. Accessed August 4, 2020

16 Jones NK, Rivett L, Sparkes D, et al; CITIID-NIHR COVID-19 BioResource Collaboration. Effective control of SARS-CoV-2 transmission between healthcare workers during a period of diminished community prevalence of COVID-19. eLife 2020;9:e59391 